Tom 25 № 1

УДК 517.982.1, 517.988.6

\title{
О СУЩЕСТВОВАНИИ И ОЦЕНКАХ РЕШЕНИЙ ФУНКЦИОНАЛЬНЫХ ВКЛЮЧЕНИЙ ${ }^{1}$
}

\author{
Е. С. Жуковский, Е. М. Якубовская
}

\begin{abstract}
Рассмотрены вопросы разрешимости операторных включений в частично упорядоченных пространствах. Используется понятие упорядоченного накрывания многозначных отображений, предложенное А. В. Арутюновым, Е.С. Жуковским и С.Е. Жуковским в 2016 г. (см. Topology Appl., 2016, vol. 201, pp. 330-343). Доказано утверждение о сохранении свойства упорядоченного накрывания при антитонных возмущениях. Получены условия упорядоченного накрывания многозначного оператора Немыцкого, действующего из пространства существенно ограниченных функций в пространство измеримых функций. А именно установлено, что если многозначное отображение $f(t, x)$ по второму аргументу является упорядоченно накрывающим (в пространстве $\mathbb{R}^{n}$ ), то соответствующий оператор Немыцкого (определяемый как множество измеримых сечений отображения $f(t, x(t)))$ также является упорядоченно накрывающим. На основании полученных результатов исследуется функциональное включение с отклоняющимся аргументом вида $0 \in g(t, x(h(t)), x(t))$. Предполагается, что многозначное отображение $g(t, x, y)$ по второму аргументу не возрастает, а по третьему аргументу является упорядоченно накрывающим. Для этого включения доказана теорема существования решений и получены оценки решений.
\end{abstract}

Ключевые слова: упорядоченное пространство; многозначное упорядоченно накрывающее отображение; многозначный оператор Немыцкого; пространство измеримых функций; функциональное включение; существование решения.

E. S. Zhukovskiy, E. M. Yakubovskaya. On the existence and estimates of solutions to functional equations.

We consider the issues of solvability of operator inclusions in partially ordered spaces. We use the notion of ordered covering of multivalued mappings proposed by A. V. Arutyunov, E. S. Zhukovskiy, and S. E. Zhukovskiy in their paper "Coincidence points principle for set-valued mappings in partially ordered spaces," Topology Appl. 201, 330-343 (2016). A statement on the preservation of properties of an ordered covering under antitone perturbations is proved. Conditions for an ordered covering of the multivalued Nemytskii operator acting from the space of essentially bounded functions to the space of measurable functions are obtained. More exactly, it is established that, if the multivalued mapping $f(t, x)$ is orderly covering in the second argument (in the space $\mathbb{R}^{n}$ ), then the corresponding Nemytskii operator (defined as the set of measurable sections of the mapping $f(t, x(t))$ ) is also orderly covering. These results are used to study a functional inclusion with a deviating argument of the form $0 \in g(t, x(h(t)), x(t))$. It is assumed that the multivalued mapping $g(t, x, y)$ is nonincreasing in the second argument and is orderly covering in the third argument. For this inclusion, a solution existence theorem is proved and estimates of solutions are obtained.

Keywords: ordered space, multivalued orderly covering mapping, multivalued Nemytskii operator, space of measurable functions, functional inclusion, existence of a solution.

MSC: 47H07, 54C60, 34K09, 55M20

DOI: $10.21538 / 0134-4889-2019-25-1-45-54$

\section{Введение}

Регулярные (накрывающие) отображения метрических пространств изучаются многими авторами и используются для решения различных задач (см. [1-3] и библиографию этих работ). В работах $[4 ; 5]$ предложен аналог понятия накрывания для отображений упорядоченных пространств, исследована задача о точках совпадения упорядоченно накрывающего и изотонного отображений. Кроме того, авторами показано, что известные результаты о точках

\footnotetext{
${ }^{1}$ Работа выполнена при финансовой поддержке Минобрнауки России (задание № 3.8515.2017/БЧ), Российского фонда фундаментальных исследований (проекты № 17-51-12064, № 17-01-00553).
} 
совпадения отображений в метрических пространствах (см., например [1]) могут быть получены из соответствующих результатов в упорядоченных пространствах. Таким образом, анализ накрывающих отображений упорядоченных пространств может дать эффективные инструменты исследования различных задач. Развитию теории таких отображений и ее приложениям посвящены исследования [6-9]. В [6;7] получены утверждения об операторных уравнениях с упорядоченно накрывающими отображениями, на основании которых для дифференциального и интегрального уравнений неявного вида доказан аналог теоремы Чаплыгина о неравенстве. Статьи [8;9] посвящены распространению методов анализа накрывающих отображений на операторные и функциональные включения. В [8] сформулированы условия устойчивости упорядоченного накрывания многозначного отображения к антитонным возмущениям. В [9] сформулировано утверждение о существовании измеримого решения функционального включения. Это утверждение является реализацией теоремы об устойчивости упорядоченного накрывания к антитонным возмущениям для многозначного отображения конкретного вида в пространстве измеримых функций. В данной статье мы доказываем результаты, анонсированные в $[8 ; 9]$, а также исследуем свойство упорядоченного накрывания многозначного оператора Немыцкого.

\section{1. Антитонные возмущения упорядоченно накрывающих многозначных отображений}

Частично упорядоченное пространство, т. е. множество $X$ с заданным на нем порядком $\preceq$, обозначаем через $X=(X, \preceq)$; используем также обозначения $x \succeq u$ в случае, если $u \preceq x$, и $x \prec u$ или $u \succ x$, если $x \preceq u, x \neq u$. Для элементов $u, v \in X$ и множества $U \subset X$ будем обозначать

$$
\begin{gathered}
\mathcal{O}_{X}(u) \doteq\{x \in X: x \preceq u\}, \quad \mathcal{O}_{X}(U) \doteq \bigcup_{u \in U} \mathcal{O}_{X}(u), \\
\mathcal{D}_{X}(u) \doteq\{x \in X: x \succeq u\}, \quad[v, u]_{X} \doteq\{x \in X: v \preceq x \preceq u\} .
\end{gathered}
$$

Пусть заданы пространства $(X, \preceq),(Y, \preceq)$. Под многозначным отображением $F: X \rightrightarrows Y$ понимаем отображение, сопоставляющее каждому $x \in X$ непустое множество $F(x) \subset Y$. Многозначное отображение $F: X \rightrightarrows Y$ называют антитонным на множестве $V \subset X$, если

$$
\forall x, x^{\prime} \in V \quad x^{\prime} \preceq x \quad \forall y \in F(x) \quad \exists y^{\prime} \in F\left(x^{\prime}\right) \quad y^{\prime} \succeq y .
$$

В случае $V=X$ антитонное на всем пространстве $X$ отображение называют антитонным (не упоминая множество $X)$.

В [5] определено следующее свойство многозначных отображений.

О п р е д е л е н и е 1 . Говорим, что отображение $F: X \rightrightarrows Y$ упорядоченно накрывает (является упорлдоченно накрывающим) множество $W \subset Y$, если

$$
\forall x \in X \quad \mathcal{O}_{Y}(F(x)) \cap W \subset F\left(\mathcal{O}_{X}(x)\right) .
$$

Слово "упорядоченно" далее будем опускать, т. е. будем говорить, что отображение $F$ накрываeт множество $W$. Отображение, накрывающее все пространство $Y$, называем накрывающим.

Заметим, что отображение $F$ накрывает множество $W$ тогда и только тогда, когда справедливо следующее соотношение

$$
\forall x \in X \quad \forall y \in F(x) \quad \forall y^{\prime} \in W \quad y^{\prime} \preceq y \quad \Rightarrow \quad \exists x^{\prime} \in X \quad x^{\prime} \preceq x, \quad y^{\prime} \in F\left(x^{\prime}\right) .
$$

Рассмотрим задачу об устойчивости свойства накрывания при антитонных возмущениях. Пусть задано отображение $\Upsilon: X^{2} \rightrightarrows Y$, которое по первому аргументу является накрывающим 
некоторое множество $W$. Устойчивость свойства накрывания означает, что если по второму аргументу отображение $\Upsilon$ антитонное, то отображение $F: X \rightrightarrows Y$, определенное соотношением

$$
F(x) \doteq \Upsilon(x, x) \quad \forall x \in X
$$

также будет накрывающим $W$. Для однозначных отображений утверждения об устойчивости свойства накрывания при антитонных возмущениях получены в работах [6;7].

Подобный подход к проблеме устойчивости накрывания в метрических пространствах был предложен в [2;3], где рассматривалось однозначное отображение $\Upsilon$ двух аргументов, являющееся по первому из них (метрически) $\alpha$-накрывающим, а по второму $\beta$-липшицевым, и утверждалось, что при $\alpha>\beta$ отображение $F$ будет $(\alpha-\beta)$-накрывающим. Соответствующий результат для многозначных отображений метрических пространств получен в [10]. Такая трактовка устойчивости свойства накрывания является естественным обобщением подхода к этой проблеме в линейных пространствах: известная теорема Милютина [11] о липшицевых возмущениях накрывающего однозначного отображения, действующего в линейное метрическое пространство, утверждает, что разность $\alpha$-накрывающего и $\beta$-липшицева отображений в случае $\alpha>\beta$ является $(\alpha-\beta)$-накрывающим отображением.

По отображению $\Upsilon: X^{2} \rightrightarrows Y$ и элементу $y^{\prime} \in Y$ определим множество $\mathcal{S}_{X}\left(\Upsilon, y^{\prime}\right)$ всех цепей $S \subset X$ таких, что

$$
\begin{gathered}
\forall x \in S \quad \exists y \in \Upsilon(x, x) \quad y \succeq y^{\prime}, \\
\forall x_{1}, x_{2} \in S \quad x_{2} \prec x_{1} \Rightarrow \exists \widetilde{x}_{2}, \widetilde{x}_{1} \in\left[x_{2}, x_{1}\right]: \widetilde{x}_{2} \preceq \widetilde{x}_{1}, \quad y^{\prime} \in \Upsilon\left(x_{2}, \widetilde{x}_{2}\right), \quad y^{\prime} \in \Upsilon\left(\widetilde{x}_{1}, x_{1}\right) .
\end{gathered}
$$

Теорема 1. Пусть при любом $x \in X$ отображсене $\Upsilon(\cdot, x): X \rightrightarrows Y$ накрывает множество $W \subset Y$; отображение $\Upsilon(x, \cdot): X \rightrightarrows Y$ является антитонным на множестве $\mathcal{D}_{X}(x)$; для любого $y^{\prime} \in W$ любая иепь $S \in \mathcal{S}_{X}\left(\Upsilon, y^{\prime}\right)$ ограничена снизу и имеет нижнюю граничу $\omega \in X$ такую, что существует элемент $y \in \Upsilon(\omega, \omega)$, удовлетворлющий неравенству $y \succeq y^{\prime}$. Тогда определенное соотношением (1.2) отображение $F: X \rightrightarrows Y$ накрывает множество $W$.

Это утверждение уточняет результат, полученный в [8, теорема 1] (в части требований к входящим в совокупность $\mathcal{S}_{X}\left(\Upsilon, y^{\prime}\right)$ цепям). Отметим также, что цитируемый результат сформулирован в [8] без доказательства.

Д о к а з а т е л ь с т в о. Покажем, что для определенного формулой (1.2) отображения $F$ справедливо соотношение (1.1).

Пусть для произвольных $y^{\prime} \in W$ и $x_{0} \in X$ существует $y_{0} \in F\left(x_{0}\right)$ такой, что $y_{0} \succeq y^{\prime}$.

Определим множество

$$
U=\left\{x \in X: \exists y \in \Upsilon(x, x) \quad y \succeq y^{\prime}\right\} \subset X .
$$

Это множество не пусто, $x_{0} \in U$. Определим в $U$ порядок $\unlhd$ следующим образом:

$$
\begin{gathered}
\forall x_{1} x_{2} \in U \quad x_{2} \triangleleft x_{1} \Leftrightarrow \\
x_{2} \prec x_{1} \text { и } \exists \widetilde{x}_{2}, \widetilde{x}_{1} \in\left[x_{2}, x_{1}\right]: \widetilde{x}_{2} \preceq \widetilde{x}_{1}, \quad y^{\prime} \in \Upsilon\left(x_{2}, \widetilde{x}_{2}\right), \quad y^{\prime} \in \Upsilon\left(\widetilde{x}_{1}, x_{1}\right) .
\end{gathered}
$$

Очевидно, что для бинарного отношения $\unlhd$ выполняются условия рефлексивности и антисимметричности; проверим транзитивность. Пусть $x_{2} \triangleleft x_{1}, x_{3} \triangleleft x_{2}$ (случай $x_{2}=x_{1}$ или $x_{3}=x_{2}$ не рассматриваем вследствие очевидности соотношения $x_{3} \unlhd x_{1}$ ). Тогда $x_{3} \prec x_{2} \prec x_{1}$ и существуют такие элементы $\widetilde{x}_{3} \in\left[x_{3}, x_{2}\right], \widetilde{x}_{1} \in\left[x_{2}, x_{1}\right]$, что выполнены включения $y^{\prime} \in \Upsilon\left(x_{3}, \widetilde{x}_{3}\right)$, $y^{\prime} \in \Upsilon\left(\widetilde{x}_{1}, x_{1}\right)$. Таким образом, $x_{3} \unlhd x_{1}$.

Согласно теореме Хаусдорфа в пространстве $(U, \unlhd)$ существует максимальная цепь $S$, которая содержит точку $x_{0}$. Если для некоторого $x \in S$ выполнено $y^{\prime} \in \Upsilon(x, x)$, то соотношение (1.1) справедливо. Пусть $y^{\prime} \notin \Upsilon(x, x)$ при любом $x \in S$. Тогда для каждого $x \in S$, так как 
отображение $\Upsilon(\cdot, x)$ накрывает множество $W$, существует $x^{\prime} \prec x$ такой, что $y^{\prime} \in \Upsilon\left(x^{\prime}, x\right)$, а в силу антитонности на множестве $\mathcal{D}_{X}\left(x^{\prime}\right)$ отображения $\Upsilon\left(x^{\prime}, \cdot\right)$ существует $\widetilde{y} \in \Upsilon\left(x^{\prime}, x^{\prime}\right)$, удовлетворяющий неравенству $\widetilde{y} \succeq y^{\prime}$. Аналогично устанавливается существование таких элементов $x^{\prime \prime} \prec x^{\prime}, \bar{y} \in \Upsilon\left(x^{\prime \prime}, x^{\prime \prime}\right)$, что $y^{\prime} \in \Upsilon\left(x^{\prime \prime}, x^{\prime}\right), \bar{y} \succeq y^{\prime}$. Таким образом, $x^{\prime \prime} \in U$ и $x^{\prime \prime} \triangleleft x$. Поскольку цепь $S$ максимальная, в ней есть элемент $\widetilde{x} \triangleleft x$ (не обязательно $\widetilde{x}=x^{\prime \prime}$ ).

Цепь $S$ принадлежит совокупности $\mathcal{S}_{X}\left(\Upsilon, y^{\prime}\right)$, и в силу соответствующего предположения теоремы относительно исходного порядка $\preceq$ она имеет нижнюю границу $\omega \in X$, причем существует элемент $y \in \Upsilon(\omega, \omega)$, удовлетворяющий неравенству $y \succeq y^{\prime}$.

Вследствие накрывания отображением $\Upsilon(\cdot, \omega)$ множества $W$ существует $z \in X$ такой, что

$$
y^{\prime} \in \Upsilon(z, \omega), \quad z \preceq \omega .
$$

Из условия антитонности на множестве $\mathcal{D}_{X}(z)$ отображения $\Upsilon(z, \cdot)$ следует существование элемента $y^{\prime \prime} \in \Upsilon(z, z)$ такого, что $y^{\prime \prime} \succeq y^{\prime}$. Таким образом, $z \in U$.

Для любого $x \in S$, как показано выше, существует $\widetilde{x} \in S, \widetilde{x} \triangleleft x$. Поэтому для некоторого $\widetilde{x}_{1} \in[\widetilde{x}, x]$ справедливо $y^{\prime} \in \Upsilon\left(\widetilde{x}_{1}, x\right)$. Из этого включения и из соотношения (1.3) следует неравенство $z \unlhd x$. В силу максимальности цепи $S$ имеем $z \in S$, тогда $z \unrhd \omega$. Но в то же время $z \unlhd \omega$, а это значит, что $z=\omega$. Из (1.3) получаем $y^{\prime} \in \Upsilon(z, z)$.

Отметим, что теорема 1 является многозначным аналогом утверждений [6;7] об устойчивости свойства накрывания однозначных отображений при антитонных возмущениях.

\section{2. Вспомогательные сведения о пространстве измеримых функций}

Для применения теоремы 1 к неявным функциональным включениям с отклоняющимся аргументом нам потребуются свойства линейно упорядоченных множеств в пространствах измеримых функций и условия (упорядоченного) накрывания многозначного оператора Немыцкого, действующего в пространствах измеримых функций.

Будем пользоваться следующими обозначениями:

$\mathbb{R}^{n}$ - пространство действительных векторов $\eta=\left(\eta_{1}, \ldots, \eta_{n}\right)$;

$|\eta|_{\mathbb{R}^{n}}=\sum_{i=1}^{n}\left|\eta_{i}\right| ;$

$\mathbb{R}_{+}^{n}$ - конус векторов из $\mathbb{R}^{n}$ с неотрицательными компонентами;

$\mu$ - мера Лебега на $[a, b]$;

$M \doteq M\left([a, b], \mathbb{R}^{n}\right)-$ пространство измеримых (по Лебегу) функций $x:[a, b] \rightarrow \mathbb{R}^{n} ;$

$L_{\infty} \doteq L_{\infty}\left([a, b], \mathbb{R}^{n}\right), L_{1} \doteq L_{1}\left([a, b], \mathbb{R}^{n}\right)$ - пространства измеримых существенно ограниченных и, соответственно, суммируемых (по Лебегу) функций $x:[a, b] \rightarrow \mathbb{R}^{n}$, с нормами

$$
\|x\|_{L_{\infty}}=\operatorname{vrai} \sup _{t \in[a, b]}|x(t)|_{\mathbb{R}^{n}}, \quad\|x\|_{L_{1}}=\int_{a}^{b}|x(s)|_{\mathbb{R}^{n}} d s .
$$

Для множества $B \subset \mathbb{R}^{n}$ определим подмножество $L_{\infty}(B) \doteq L_{\infty}([a, b], B)$ пространства $L_{\infty}$, содержащее функции со значениями $x(t) \in B \dot{\forall} t \in[a, b]$ (здесь и ниже символ $\dot{\forall}$ означает "при почти всех"). Если множество $B$ не пусто, то, очевидно, $L_{\infty}(B)$ также не пусто и справедливы вложения $L_{\infty}(B) \subset L_{\infty} \subset L_{1} \subset M$.

В рассматриваемых пространствах измеримых функций определим порядок, полагая для их элементов $x, u$ выполненным неравенство $x \leq u$, если $x(t) \leq u(t) \dot{\forall} t \in[a, b]$.

Известно, что любая ограниченная снизу цепь $S$ измеримых функций имеет точную нижнюю границу inf $S \in M$ (см., например, [12, с. 706]). Нам потребуется не только существование, но и следующее свойство инфимума. 
Лемма 1. Пусть множество $B \subset \mathbb{R}^{n}$ компактно. Тогда для любой цепи $S \subset L_{\infty}(B)$ существует точная нижнял граница $w \doteq \inf S \in L_{\infty}(B)$, и если иепь $S$ бесконечна $и w \notin S$, то существует такая убывающая последовательность ее элементов $\left\{u_{i}\right\} \subset S$, что

$$
w(t)=\inf \left\{u_{i}(t)\right\}=\lim _{i \rightarrow \infty} u_{i}(t) \quad \dot{\forall} t \in[a, b] .
$$

Д о к а з а т е л ь с т в о. Существование инфимума конечной цепи очевидно. Будем предполагать, что цепь $S \subset L_{\infty}(B)$ бесконечна.

Вначале заметим, что для любых двух функций $x, u \in L_{\infty}$, если $x>u$, то существует такое $\delta>0$, что при некотором $i=\overline{1, n}$ для $i$-х компонент $x_{i}, u_{i}$ функций $x, u$ справедливо

$$
\mu\left\{t \in[a, b]: x_{i}(t) \geq u_{i}(t)+\delta\right\}>0 .
$$

Действительно, в противном случаем при любых $i=\overline{1, n}, k=1,2, \ldots$ выполнено $x_{i}(t)<$ $u_{i}(t)+k^{-1} \dot{\forall} t \in[a, b]$; отсюда получаем неравенство $x(t) \leq u(t) \dot{\forall} t \in[a, b]$, противоречащее исходному неравенству $x>u$.

Из оценки (2.1) следует, что для цепи $S \subset L_{\infty}(B)$ множество

$$
I_{S} \doteq\left\{\int_{a}^{b} u(s) d s: u \in S\right\} \subset \mathbb{R}^{n}
$$

является ограниченной цепью в $\mathbb{R}^{n}$ и для любых $x, u \in S, x>u$, выполнено неравенство

$$
\int_{a}^{b} x(s) d s>\int_{a}^{b} u(s) d s .
$$

Определим $\eta \doteq \inf I_{S}$. Если для некоторого элемента $z \in S$ окажется, что $\int_{a}^{b} z(s) d s=\eta$, то $z=\inf S$.

Пусть $\int_{a}^{b} u(s) d s \neq \eta$ для любого $u \in S$. В этом случае найдем убывающую последовательность функций $u^{k} \in S, k=1,2, \ldots$, для которой убывающая последовательность векторов

$$
\eta^{k} \doteq \int_{a}^{b} u^{k}(s) d s \in \mathbb{R}^{n}
$$

сходится к $\eta$. Относительно метрики пространства $L_{1}$ последовательность функций $u^{k}$ является фундаментальной, поскольку $\left\|u^{k}-u^{k^{\prime}}\right\|_{L_{1}}=\left|\eta^{k}-\eta^{k^{\prime}}\right|_{\mathbb{R}^{n}}$ при любых натуральных $k, k^{\prime}$. Следовательно, существует $w=\lim _{k \rightarrow \infty} u^{k}$, т. е. $\left\|u^{k}-w\right\|_{L_{1}} \rightarrow 0$. Из сходящейся в $L_{1}$ последовательности можно извлечь подпоследовательность, сходящуюся почти всюду; значит и сама убывающая последовательность $\left\{u^{k}\right\}$ сходится к $w$ почти всюду; таким образом, $w(t)=\lim _{k \rightarrow \infty} u^{k}(t) \dot{\forall} t \in[a, b]$. Так как множество $B$ замкнуто, то $w(t) \in B \dot{\forall} t \in[a, b]$. Поскольку множество $B$ ограничено, $w \in L_{\infty}(B)$. В силу убывания последовательности $\left\{u^{k}(t)\right\}$ имеем $w(t)=\inf \left\{u^{k}(t)\right\} \dot{\forall} t \in[a, b]$.

Для любого $u \in S$, так как $\int_{a}^{b} u(s) d s>\eta$, существует номер $k$, при котором $\int_{a}^{b} u(s) d s>\eta^{k}$ и, следовательно, $u>u^{k}>w$. Таким образом, $w$ является нижней границей $S$. Так как $w$ еще и точная нижняя граница $\left\{u^{k}\right\} \subset S$, то $w=\inf S$.

Нам потребуется следующий, достаточно очевидный, критерий существования неотрицательных селекторов измеримого отображения. 
Лемма 2. Пусть задано измеримое многозначное отображение $\varphi:[a, b] \rightrightarrows \mathbb{R}^{m}$ c замкнутыми значениями. Для существования у отображения $\varphi$ неотрицательного селектора ( такой измеримой функиии $y:[a, b] \rightarrow \mathbb{R}^{m}$, что $\left.y(t) \in \varphi(t), y(t) \geq 0 \dot{\forall} t \in[a, b]\right)$, необходимо и достаточно, чтобы $\varphi(t) \cap \mathbb{R}_{+}^{m} \neq \varnothing \dot{\forall} t \in[a, b]$.

Д о к а з а т е л ь с т в о. В силу измеримости отображения $\varphi$ множество

$$
T \doteq\left\{t \in[a, b]: \varphi(t) \cap \mathbb{R}_{+}^{m} \neq \varnothing\right\}
$$

измеримо. Определим множество $\bar{T} \doteq[a, b] \backslash T$. Для любого селектора $y:[a, b] \rightarrow \mathbb{R}^{m}$ отображения $\varphi$, при $t \in \bar{T}$, очевидно, имеем $y(t) \ngtr 0$. Таким образом, если $\mu(\bar{T})>0$, то у отображения $\varphi$ нет неотрицательного селектора.

Пусть $\mu(\bar{T})=0$, тогда при почти всех $t \in[a, b]$ множество $\varphi(t) \cap \mathbb{R}_{+}^{m}$ не пусто и замкнуто. Отображение $\Phi \doteq \varphi \cap \mathbb{R}_{+}^{m}:[a, b] \rightrightarrows \mathbb{R}^{m}$ измеримо. Действительно, для любого замкнутого множества $\Omega \subset \mathbb{R}^{m}$ его полный прообраз

$$
\Phi_{-}^{-1}(\Omega) \doteq\left\{t \in[a, b]:\left(\varphi(t) \cap \mathbb{R}_{+}^{m}\right) \cap \Omega \neq \varnothing\right\}=\left\{t \in[a, b]: \varphi(t) \cap\left(\mathbb{R}_{+}^{m} \cap \Omega\right) \neq \varnothing\right\}
$$

есть измеримое множество (это прямо следует из измеримости отображения $\varphi$ ). Остается заметить, что любой измеримый селектор отображения $\Phi$ является неотрицательным селектором отображения $\varphi$.

Теперь сформулируем условия накрывания многозначного оператора Немыцкого в пространствах измеримых функций.

Рассмотрим многозначную функцию $f:[a, b] \times \mathbb{R}^{n} \rightrightarrows \mathbb{R}^{m}$ со значениями - замкнутыми множествами $f(t, x) \subset \mathbb{R}^{m}$. Пусть эта функция удовлетворяет условиям Каратеодори, т. е. $f(\cdot, x):[a, b] \rightrightarrows \mathbb{R}^{m}$ измерима, а $f(t, \cdot):[a, b] \rightrightarrows \mathbb{R}^{m}$ непрерывна по Хаусдорфу. Данные предположения позволяют определить многозначный оператор Немыцкого $N_{f}: L_{\infty} \rightrightarrows M$ равенством

$$
\forall x \in L_{\infty} N_{f} x=\{y \in M: y(t) \in f(t, x(t)) \dot{\forall} t \in[a, b]\} .
$$

Установим связь между свойствами накрывания функции $f$ по второму аргументу и многозначного оператора Немыцкого $N_{f}$.

Пусть задано $r>0$. Обозначим через $f_{r}$ сужение функции $f$ на множество $[a, b] \times B_{r}$, где $B_{r} \doteq\left\{x \in \mathbb{R}^{n}:|x|_{\mathbb{R}^{n}} \leq r\right\}$ - шар в $\mathbb{R}^{n}$ с центром в 0 и радиуса $r$. Определим соответствующий многозначный оператор Немыцкого

$$
N_{f_{r}}: L_{\infty}\left(B_{r}\right) \rightrightarrows M, \quad \forall x \in L_{\infty}\left(B_{r}\right) N_{f_{r}}=\left\{y \in M: y(t) \in f_{r}(t, x(t)) \dot{\forall} t \in[a, b]\right\} .
$$

Пусть задано измеримое многозначное отображение $V:[a, b] \rightrightarrows \mathbb{R}^{m}$ с замкнутыми значениями. Определим множество

$$
\operatorname{Sel}[V] \doteq\{y \in M: y(t) \in V(t) \dot{\forall} t \in[a, b]\} .
$$

Лемма 3. Если при почти всех $t \in[a, b]$ функиия $f_{r}(t, \cdot): B_{r} \rightrightarrows \mathbb{R}^{m}$ накрывает множество $V(t) \subset \mathbb{R}^{m}$, то оператор Немыцкого $N_{f_{r}}: L_{\infty}\left(B_{r}\right) \rightrightarrows M$ накрывает множество $\operatorname{Sel}[V] \subset M$.

Д о к а з а т е л ь с т в о. Пусть $y \in \operatorname{Sel}[V]$ и задан элемент $u \in L_{\infty}\left(B_{r}\right)$ такой, что существует $z \in N_{f_{r}}(u), z \geq y$. Эти соотношения означают, что $z(t) \in f_{r}(t, u(t))$ и $z(t) \geq y(t)$ почти всюду на $[a, b]$. В силу накрывания функции $f_{r}$ по второму аргументу выполнено

$$
y(t) \in f_{r}(t, U(t)) \dot{\forall} t \in[a, b],
$$

где множество $U(t) \doteq\left\{x \in B_{r}: x \leq u(t)\right\}$, очевидно, не пусто, а отображение $U:[a, b] \rightrightarrows \mathbb{R}^{n}$ измеримо. 
Согласно лемме Филиппова (см., например, [13, теорема 1.5.15] - для случая компактнозначной функции $f_{r}$ и [14, следствие 3$]$ - для функции $f_{r}$ с замкнутыми значениями) существует такая измеримая функция $\bar{x}:[a, b] \rightarrow \mathbb{R}^{n}$, что

$$
\bar{x}(t) \in U(t), \quad y(t) \in f_{r}(t, \bar{x}(t)) \quad \dot{\forall} t \in[a, b] .
$$

Таким образом, имеют место включения $y \in N_{f_{r}}(\bar{x})$ и $\bar{x} \in M\left(B_{r}\right)$. Следовательно, отображение $N_{f_{r}}$ накрывает множество $\operatorname{Sel}[V] \subset M$.

\section{3. Существование и оценки решений функциональных включений}

Пусть задано отображение $g:[a, b] \times \mathbb{R}^{n} \times \mathbb{R}^{n} \rightrightarrows \mathbb{R}^{m}$, имеющее замкнутые значения и удовлетворяющее условиям Каратеодори, т. е. измеримое по первому аргументу, непрерывное по второму и по третьему аргументам; задана измеримая функция $h:[a, b] \rightarrow[a, b]$ такая, что для любого измеримого множества $E \subset[a, b]$ множество $h^{-1}(E)$ измеримо и если $\mu(E)=0$, то $\mu\left(h^{-1}(E)\right)=0$. Рассмотрим функциональное включение

$$
0 \in g(t, x(h(t)), x(t)), \quad t \in[a, b] .
$$

Получим условия существования его решения в классе существенно ограниченных функций $x:[a, b] \rightarrow \mathbb{R}^{n}$. Пусть $r>0, B_{r} \doteq\left\{x \in \mathbb{R}^{n}:|x|_{\mathbb{R}^{n}} \leq r\right\}$. Определим сужение $g_{r}:[a, b] \times B_{r} \times B_{r} \rightrightarrows$ $\mathbb{R}^{m}$ отображения $g$.

Теорема 2. Пусть для некоторой функиии $u_{0} \in L_{\infty}\left(B_{r}\right)$ при почти всех $t \in[a, b]$ множество $g_{r}\left(t, u_{0}(h(t)), u_{0}(t)\right) \cap \mathbb{R}_{+}^{m}$ не пусто. Пусть при почти всех $t \in[a, b]$ и любом $x \in B_{r}$ отображсение $g_{r}(t, x, \cdot): B_{r} \rightrightarrows \mathbb{R}^{m}$ накрывает множество $\{0\} \subset \mathbb{R}^{m}$, отображсение $g_{r}(t, \cdot, x): B_{r} \rightrightarrows$ $\mathbb{R}^{m}$ - антитонное. Тогда существует решение $x \in L_{\infty}\left(B_{r}\right)$ включения (3.2), удовлетворяющее неравенству $x(t) \leq u_{0}(t)$ при почти всех $t \in[a, b]$.

Д о к а з а т е л ь с т в о. Определим оператор Немыцкого $N_{g_{r}}: L_{\infty}\left(B_{r}\right) \times L_{\infty}\left(B_{r}\right) \rightrightarrows M$ соотношением

$$
\forall x_{1}, x_{2} \in L_{\infty}\left(B_{r}\right) \quad N_{g_{r}}\left(x_{1}, x_{2}\right)=\left\{y \in M: y(t) \in g_{r}\left(t, x_{2}(t), x_{1}(t)\right) \dot{\forall} t \in[a, b]\right\} .
$$

Обозначим $(H x)(t)=x(h(t))$. В силу сделанных предположений для любой измеримой функции $x(\cdot)$ функция $(H x)(\cdot)$ измерима (см. $\left[12\right.$, с. 706]) и $H: L_{\infty}\left(B_{r}\right) \rightarrow L_{\infty}\left(B_{r}\right)$. Включение $(3.2)$ запишем в виде $0 \in N_{g_{r}}(x, H x)$. Используя теорему 1 , покажем, что отображение $F: L_{\infty}(B) \rightrightarrows$ $M, F(x) \doteq N_{g_{r}}(x, H x)$ накрывает множество $\{0\} \subset M$.

Из леммы 3 следует, что для любого $u \in L_{\infty}\left(B_{r}\right)$ отображение $N_{g_{r}}(\cdot, u): L_{\infty}\left(B_{r}\right) \rightrightarrows M$ накрывает множество $\{0\} \subset M$.

Так как отображение $g_{r}(t, \cdot, x): B_{r} \rightrightarrows \mathbb{R}^{m}$ при любом $x \in \mathbb{R}^{n}$ и почти всех $t \in[a, b]$ антитонное, то, очевидно, отображение $N_{g_{r}}(u, H(\cdot)): L_{\infty}\left(B_{r}\right) \rightrightarrows M$ также является антитонным при любом $u \in L_{\infty}\left(B_{r}\right)$.

Рассмотрим произвольную цепь $S \subset L_{\infty}\left(B_{r}\right)$ такую, что для любого $x \in S$ существует неотрицательный $y \in N_{g_{r}}(x, H x)$ и для любых $x_{1}, x_{2} \in S, x_{2}<x_{1}$, существуют $\widetilde{x}_{2}, \widetilde{x}_{1} \in L_{\infty}\left(B_{r}\right)$, удовлетворяющие соотношениям

$$
x_{2} \leq \widetilde{x}_{2} \leq \widetilde{x}_{1} \leq x_{1}, \quad 0 \in N_{g_{r}}\left(x_{2}, H \widetilde{x}_{2}\right), \quad 0 \in N_{g_{r}}\left(\widetilde{x}_{1}, H x_{1}\right)
$$

Эта цепь ограничена, и у нее существует точная нижняя граница, обозначим $\omega \doteq \inf S$. Если $\omega \in S$, то, очевидно, в множестве $N_{g_{r}}(w, H w)$ найдется неотрицательная функция. Пусть $\omega \notin S$. Согласно лемме 1 существует такая убывающая последовательность $\left\{x_{i}\right\} \subset S$, что $\inf \left\{x_{i}\right\}=\omega$, 
$\lim _{i \rightarrow \infty} x_{i}(t)=\omega(t) \dot{\forall} t \in[a, b]$. По определению цепи $S$ при каждом $i$ существует $\widetilde{x}_{i} \in\left[x_{i+1}, x_{i}\right]$ такой, что $0 \in N_{g_{r}}\left(\widetilde{x}_{i}, H x_{i}\right)$, т. е. почти всюду на $[a, b]$ выполнено $0 \in g_{r}\left(t, x_{i}(h(t)), \widetilde{x}_{i}(t)\right)$. Имеем $\lim _{i \rightarrow \infty} \widetilde{x}_{i}(t)=\omega(t) \dot{\forall} t \in[a, b]$. В силу непрерывности функции $g_{r}(t, \cdot, \cdot)$ получаем включение

$$
0 \in g_{r}(t, \omega(h(t)), \omega(t)) \quad \dot{\forall} t \in[a, b] .
$$

Таким образом, $0 \in N_{g_{r}}(\omega, H \omega)$.

Итак, согласно теореме 1 отображение $F: L_{\infty}(B) \rightrightarrows M, F(x) \doteq N_{g_{r}}(x, H x)$ накрывает множество $\{0\} \subset M$. Далее, из предположения

$$
g_{r}\left(t, u_{0}(h(t)), u_{0}(t)\right) \cap \mathbb{R}_{+}^{m} \neq \varnothing \quad \dot{\forall} t \in[a, b]
$$

в силу леммы 2 следует, что существует неотрицательная функция $y_{0} \in M$ такая, что $y_{0} \in F\left(u_{0}\right)$. А так как $F$ накрывает множество $\{0\} \subset M$, существует решение $x \in L_{\infty}(B)$ включения (3.5), удовлетворяющее при почти всех $t \in[a, b]$ неравенству $x(t) \leq u_{0}(t)$.

\section{СПИСОК ЛИТЕРАТУРЫ}

1. Арутюнов А.В. Накрывающие отображения в метрических пространстввах и неподвижные точки // Докл. АН. 2007. Т. 416, № 2. С. 151-155.

2. Аваков Е.Р., Арутюнов А.В., Жуковский Е.С. Накрывающие отображения и их приложения к дифференциальным уравнениям, не разрешенным относительно производной // Дифференц. уравнения. 2009. Т. 45, № 5. С. 613-634.

3. Arutyunov A.V., Zhukovskiy E.S., Zhukovskiy S.E. Covering mappings and well-posedness of nonlinear Volterra equations // Nonlinear Analysis: Theory, Methods and Applications. 2012. Vol. 75, iss. 3. P. 1026-1044. doi: 10.1016/j.na.2011.03.038.

4. Arutyunov A.V., Zhukovskiy E.S., Zhukovskiy S.E. Coincidence points principle for mappings in partially ordered spaces // Topology Appl. 2015. Vol. 179, № 1. P. 13-33. doi: $10.1016 /$ j.topol.2014.08.013 .

5. Arutyunov A.V., Zhukovskiy E.S., Zhukovskiy S.E. Coincidence points principle for setvalued mappings in partially ordered spaces // Topology Appl. 2016. Vol. 201. P. 330-343. doi: $10.1016 /$ j.topol.2015.12.044 .

6. Жуковский Е.С. Об упорядоченно накрывающих отображениях и неявных дифференциальных неравенствах // Дифференц. уравнения. 2016. Т. 52, № 12. С. 1610-1627

7. Жуковский Е.С. Об упорядоченно накрывающих отображениях и интегральных неравенствах типа Чаплыгина // Алгебра и анализ. 2018. Т. 30, № 1. С. 96-127

8. Жуковский Е.С., Плужникова Е.А., Якубовская Е.М. Об устойчивости упорядоченного накрывания многозначных отображений при антитонных возмущениях // Вестн. Тамбов. ун-та. Сер.: Естественные и технические науки. 2016. Т. 21, № 6. С. 1969-1973.

doi: 10.20310/1810-0198-2016-21-6-1969-1973.

9. Якубовская Е.М. О функциональных включениях в упорядоченных пространствах // Вестн. Тамбов. ун-та. Сер.: Естественные и технические науки. 2017. Т. 22, № 3. С. 611-614.

doi: 10.20310/1810-0198-2017-22-3-611-614 .

10. Arutyunov A., de Oliveira V.A., Pereira F. L., Zhukovskiy E., Zhukovskiy S. On the solvability of implicit differential inclusions // Applicable Analysis. 2015. Vol. 94, № 1. P. 129-143. doi: $10.1080 / 00036811.2014 .891732$.

11. Дмитрук А.В., Милютин А.А., Осмоловский Н.П. Теорема Люстерника и теория экстремума // Успехи мат. наук. 1980. Т. 35, № 6 (216). С. 11-46.

12. Данфорд Н., Шварц Дж. Линейные операторы. Т.1. Общая теория. М.: ИЛ, 1962. 896 с. ISBN: 5-354-00601-5.

13. Борисович Ю.Г., Гельман Б.Д., Мышкис А.Д., Обуховский В.В. Введение в теорию многозначных отображений и дифференциальных включений. Москва: ЛИБРОКОМ, 2011. 224 с. ISBN: 978-5-397-01526-4 . 
14. Жуковский Е.С., Панасенко Е.А. Определение метрики пространства $\operatorname{clos} \varnothing(X)$ замкнутых подмножеств метрического пространства $X$ и свойства отображений со значениями в $\operatorname{clos}_{\varnothing}\left(\mathbb{R}^{n}\right) / /$ Мат. сб. 2014. Т. 205, № 9. С. 65-96. doi: 10.4213/sm8240 .

15. Крейн С.Г. Функциональный анализ. Сер.: Справочная математическая библиотека М.: Наука, $1972.544 \mathrm{c.}$

Поступила 19.09.2018

После доработки 16.01.2019

Принята к публикации 21.01.2019

Жуковский Евгений Семенович

д-р физ.-мат. наук, профессор

директор НИИ математики, физики и информатики

Тамбовский государственный университет им. Г.Р. Державина, г. Тамбов

e-mail: zukovskys@mail.ru

Якубовская Екатерина Михайловна

аспирант кафедры функционального анализа

Тамбовский государственный университет им. Г.Р. Державина, г. Тамбов

e-mail: yak.cat1306@mail.com

\section{REFERENCES}

1. Arutyunov A.V. Covering mappings in metric spaces, and fixed points. Dokl. Math., 2007, vol. 76, no. 2, pp. 665-668. doi: 10.1134/S1064562407050079.

2. Avakov E.R., Arutyunov A.V., Zhukovskii E.S. Covering mappings and their applications to differential equations unsolved for the derivative. Differ. Equ., 2009, vol. 45, no. 5, pp. 627-649. doi: 10.1134/S0012266109050024.

3. Arutyunov A.V., Zhukovskiy E.S., Zhukovskiy S.E. Covering mappings and well-posedness of nonlinear Volterra equations. Nonlinear Analysis: Theory, Methods and Applications, 2012, vol. 75, no. 3, pp. 1026-1044. doi: 10.1016/j.na.2011.03.038.

4. Arutyunov A.V., Zhukovskiy E.S., Zhukovskiy S.E. Coincidence points principle for mappings in partially ordered spaces. Topology Appl., 2015, vol. 179, pp. 13-33. doi: 10.1016/j.topol.2014.08.013.

5. Arutyunov A.V., Zhukovskiy E.S., Zhukovskiy S.E. Coincidence points principle for set-valued mappings in partially ordered spaces. Topology Appl., 2016, vol. 201, pp. 330-343. doi: 10.1016/j.topol.2015.12.044 .

6. Zhukovskiy E.S. On ordered-covering mappings and implicit differential inequalities. Differential Equations, 2016, vol. 52, no. 12, pp. 1539-1556. doi: 10.1134/S0012266116120028.

7. Zhukovskiy E.S. On order covering maps in ordered spaces and Chaplygin-type inequalities. St. Petersburg Math. J., 2019, vol. 30, no. 1, pp. 73-94. doi: 10.1090/spmj/1530 .

8. Zhukovskiy E.S., Pluzhnikova E.A., Yakubovskaya E.M. On stability of ordered covering of multivalued mappings under antitone perturbations. Vestnik Tambovskogo Universiteta. Seriya Estestvennye i Tekhnicheskie Nauki, 2016, vol. 21, no. 6, pp. 1969-1973 (in Russian).

doi: 10.20310/1810-0198-2016-21-6-1969-1973.

9. Yakubovskaya E.M. About functional inclusions in ordered spaces. Vestnik Tambovskogo Universiteta. Seriya Estestvennye i Tekhnicheskie Nauki, 2017, vol. 22, no. 3, pp. 611-614 (in Russian). doi: 10.20310/1810-0198-2017-22-3-611-614.

10. Aram Arutyunov, Valeriano Antunes de Oliveira, Fernando Lobo Pereira, Evgeniy Zhukovskiy and Sergey Zhukovskiy. On the solvability of implicit differential inclusions. Applicable Analysis, 2015, vol. 94, no. 1, pp. 129-143. doi: 10.1080/00036811.2014.891732 .

11. Dmitruk A.V., Milyutin A.A., Osmolovskii N.P. Lyusternik's theorem and the theory of extrema. Russian Math. Surveys, 1980, vol. 35, no. 6, pp. 11-51. doi: 10.1070/RM1980v035n06ABEH001973.

12. Dunford N., Schwartz J.T. Linear operators. I. General theory. N Y, Interscience Publishers, 1958, 858 p. ISBN: 0470226056. Translated to Russian under the title Lineinye operatory. Obshchaya teoriya. Moscow: Inostr. Lit. Publ., 1962, 896 p. ISBN: 5-354-00601-5.

13. Borisovich Yu.G., Gel'man B.D., Myshkis A.D., Obukhovskii V.V. Vvedenie v teoriyu mnogoznachnykh otobrazhenii i differentsial'nykh vklyuchenii [Introduction to the theory of multi-valued mappings and differential inclusions]. Moscow: LIBROKOM Publ., 2011, 224 p. ISBN: 978-5-397-01526-4. 
14. Zhukovskiy E.S., Panasenko E.A. Definition of the metric on the space $\operatorname{clos} \varnothing(X)$ of closed subsets of a metric space $X$ and properties of mappings with values in $\operatorname{clos}_{\varnothing}\left(\mathbb{R}^{n}\right)$. Sb. Math., 2014, vol. 205, no. 9, pp. 1279-1309. doi: 10.1070/SM2014v205n09ABEH004418.

15. Vilenkin N.Ya., Krein S.G., et al. Functional analysis. Wolters-Noordhoff Series of Monographs and Textbooks on Pure and Applied Mathematics. Groningen, Wolters-Noordhoff Publ., 1972, 379 p. ISBN: 9001909809. Original Russian text published in Krein S.G. (ed.) Funkzional'nyi analiz. SMB. Moscow, Nauka Publ., 1972, 544 p.

Received September 19, 2018

Revised January 16, 2019

Accepted January 21, 2019

Funding Agency: This work was supported by the Ministry of Education and Science of the Russian Federation (state contract no. 3.8515.2017/BCh) and by the Russian Foundation for Basic Research (projects no. 17-51-12064, no. 17-01-00553).

Evgeny Semenovich Zhukovskiy, Dr. Phys.-Math. Sci., Prof., Director of the Research Institute of Mathematics, Physics and Informatics, Tambov State University named after G.R. Dergavin, Tambov, 392000 Russia, e-mail: zukovskys@mail.ru .

Ekaterina Mikhailovna Yakubovskaya, doctoral student, Tambov State University named after G.R. Dergavin, Tambov, 392000 Russia, e-mail: yak.cat1306@gmail.com . 